\title{
HCMV UL97 phosphotransferase gene mutations may be associated with antiviral resistance in immunocompromised patients in Belém, PA, Northern Brazil
}

\author{
Dorotéa de Fátima Lobato da Silva ${ }^{[1],[2], ~ J e d s o n ~ F e r r e i r a ~ C a r d o s o ~}{ }^{[3]}$, Sandro Patroca da Silva ${ }^{[3]}$, \\ Leda Mani França Arruda ${ }^{[2]}$, Renato Lopes Fernandes de Medeiros ${ }^{[2]}$, \\ Marluce Matos Moraes ${ }^{[2]}$ and Rita Catarina Medeiros Sousa ${ }^{[1]}$
}

[1]. Programa de Pós-Graduação Stricto Sensu em Patologia Tropical, Núcleo de Medicina Tropical, Universidade Federal do Pará, Belém, PA, Brasil. [2]. Laboratório de Virologia e Biologia Molecular, Seção de Meio Ambiente, Instituto Evandro Chagas, Ananindeua, PA, Brasil.

[3]. Centro de Inovações Tecnológicas, Instituto Evandro Chagas, Ananindeua, PA, Brasil.

\begin{abstract}
Introduction: Human cytomegalovirus is one of the causes of opportunist infections in immunocompromised patients, and is triggered by factors such as state of viral latency, weakened immune responses, and development of antiviral resistance to ganciclovir, the only drug offered by the public health system in Brazil to treat the infection. The goal of this study was to identify mutations that may be associated with antiviral resistance in immunocompromised patients. Methods: Molecular analysis was performed in 82 blood samples and subjected to genomic DNA extraction by a silica-based method. Three sequences of the HCMV UL97 gene, which encodes a phosphotransferase protein required for activation of ganciclovir, were amplified by polymerase chain reaction. Pyrosequencing methods were applied to one external 2096-bp segment DNA and two internal sequences between nucleotides 1087 to 1828 to detect mutations in this gene. Results: Approximately $10 \%$ of sequences contained mutations between nucleotides 377 and 594, in conserved regions of the UL97 gene, leading to amino acid changes. Eleven coding mutations were identified, including changes leading to amino acid substitutions, E596K and S604F, which were observed in $100 \%$ of samples and are described for the first time in Brazil. In addition, one mutation (A594V) that is associated with ganciclovir resistance was detected in a kidney transplant patient. Conclusions: Further studies to detect mutations associated with HCMV resistance to antiviral drugs are required to demonstrate the need to increase the variety and availability of drugs used to treat viral infections in the public health care system in Brazil.
\end{abstract}

Keywords: Cytomegalovirus. Immunocompromised. Antiviral resistance. Mutations. Phosphotransferase.

\section{INTRODUCTION}

Human cytomegalovirus (HCMV or HHV-5) is one of the main causes of opportunist infections in immunocompromised patients. Activation of the latent virus and viral resistance to drugs, along with immunodeficiency, are risk factors that contribute to increased morbidity and mortality among patients with HCMV infections ${ }^{1}$.

Several drugs are used to treat cytomegalovirus infection, although only ganciclovir is available in the public health care system in Brazil. This is a critical consideration, since many studies report the circulation of viral strains that are resistant to this drug ${ }^{2-8}$.

Corresponding author: $\mathrm{Dr}^{\mathrm{a}}$ Dorotéa Lobato

e-mail: dorotealobato@iec.pa.gov.br

Received 11 October 2017

Accepted 23 March 2018
Resistance of HCMV to antiviral drugs can be caused by mutations in one or two genes encoding the viral tegument, $U L 97$ and/or $U L 54^{9-11}$, which has been investigated by molecular methods, including restriction fragment length polymorphism (RFLP) and nucleotide sequence analysis ${ }^{7,12-14}$. The essential UL97 gene encodes the phosphotransferase enzyme pUL97 which is important for activating ganciclovir. In order to be effective, ganciclovir requires the activity of viral and cellular phosphotransferases to convert its inactive form into an active triphosphate molecule (GCV-TP), which is a powerful inhibitor of viral deoxyribonucleic acid (DNA) polymerase ${ }^{5,15,16}$. Mutations conferring resistance to ganciclovir have been detected in conserved regions of $U L 97^{5}$ and lead to generation of a defective protein, with low or no activity ${ }^{3,6,17}$. Such mutated proteins are unable to contribute to ganciclovir activation, and can lead to inadequate patient response to treatment ${ }^{18,19}$.

In Brazil, data on HCMV resistance to antiviral drugs are scarce, and most studies have been carried out in the southeast region of the country. In 2004, Carraro and Granato ${ }^{12}$ 
amplified a large region of the UL97 gene from 60 samples from kidney transplant patients; however, they did not detect any mutations associated with antiviral resistance ${ }^{12}$. In contrast, Nogueira ${ }^{7}$ described genotypes encoding important mutations, L595S (55.6\%), L595S/A594V (22.2\%), and L595F/A594V $(11.1 \%)$, associated with antiviral resistance in samples from kidney transplant patients treated with ganciclovir. Given the limited available data on this subject in Brazil, particularly the northern region, the objective of this study was to investigate mutations in HCMV UL97 gene in blood samples from immunocompromised patients, including patients with HIV, systemic lupus erythematosus patients and kidney transplant patients in the City of Belém, Pará, North Region of Brazil.

\section{METHODS}

We performed a cross-sectional, observational study with convenience sampling and active search in three public healthcare system hospitals in the City of Belém, PA, which presents with the highest number of immunocompromised patients, including 415 samples of blood collected with ethylenediaminetetraacetic acid (EDTA) anticoagulant from patients with human immunodeficiency virus/acquired immunodeficiency syndrome (HIV/AIDS) $(\mathrm{n}=264)$, systemic lupus erythematosus (SLE) $(\mathrm{n}=60)$, and renal transplant recipients $(\mathrm{n}=214)$. In total, 82 DNA samples were selected for molecular analysis, based on amplified samples of great quality, corresponding to 64 samples of HIV, 3 of SLE, 14 of renal transplant recipients and the control sample AD169 CMV strain.

\section{Ethical considerations}

The study was registered in the Sistema Nacional de Ética em Pesquisa -SISNEP (CAAE-5618.0.000.072-09) and approved by the Ethics Committee of Evandro Chagas Institute. All participants signed an informed consent form, allowing the collection of blood samples and epidemiological data.

Molecular analysis was performed for a total of 82 samples, and DNA extraction by a silica-based method, using commercial kits (GE Healthcare). DNA was amplified by the polymerase chain reaction (PCR) technique.

Mutations were identified by analyzing the nucleotide sequence of a 2096-bp fragment of the UL97 gene by pyrosequencing on a $G S$ Junior 454 platform (ROCHE) ${ }^{20}$.

The amplification reactions were performed according to the protocols published by Nogueira ${ }^{7}$ and Boivin ${ }^{21}$, using the primers CPT0 (5'-ACGGTGCTCACGGTCTGGAT-3') and CPT0 2096 CAGGCGTCGAAGCAGTGCGTGAGCTTGCCGTTCTT-3') to amplify a 2096-bp fragment ${ }^{7,21}$. Using the product of the first reaction as template, nested-PCR reactions were performed to amplify two internal fragments of $117 \mathrm{bp}$ and $531 \mathrm{bp}$. The 117-bp fragment was amplified using the primers CT1713 (5'-CGGTCTGGACGAGGTGCGCAT-3') and CT1830M GACAGGCGTCGAAGCAGTCGTGAGCTTGCGTTCTT-3'), and the 531-bp fragment with CT1088 (5'-ACGGTGCTCACGGTCTGGAT-3') and CT1619 (5'-AAACGCGCGTGCGGGTCGCAGA-3').

Reactions were performed in a final volume of $50 \mu \mathrm{L}$ and the components were as follows: $5 \mu \mathrm{L}$ of $10 \mathrm{X}$ PCR buffer, $43.15 \mu \mathrm{L}$ of distilled water, $0.6 \mathrm{mM} \mathrm{MgCl}, 0.75 \mathrm{mM}$ dNTPs, $0.1 \mathrm{pmol}$ each primer; $0.2 \mathrm{U}$ Taq DNA polymerase, $1 \%$ dimethyl sulfoxide (DMSO), and $5 \mu \mathrm{L}$ of DNA. The product amplified in the first reaction $(1 \mu \mathrm{L})$ was used as template for the nested-PCR. The reaction conditions were as follows: initial amplification, $95^{\circ} \mathrm{C}$, $4 \mathrm{~min}$, followed by 35 cycles of $95^{\circ} \mathrm{C}, 45 \mathrm{~s} ; 55^{\circ} \mathrm{C}, 30 \mathrm{~s} ; 72^{\circ} \mathrm{C}$, $90 \mathrm{~s}$; nested-PCR, $95^{\circ} \mathrm{C}, 4 \mathrm{~min}$; followed by 35 cycles of $95^{\circ} \mathrm{C}$, $30 \mathrm{~s} ; 55^{\circ} \mathrm{C}, 30 \mathrm{~s} ; 72^{\circ} \mathrm{C}, 1 \mathrm{~min}$.

The genomic library was amplified by emulsion PCR using a commercial kit (ROCHE) ${ }^{22}$. Sequence assembly was performed using Newbler software with the GS De Novo Assembler module, applying the following parameters: minimal length overlap $=40$ and minimal overlap identity $=80 \%$.

\section{RESULTS}

After amplifying the target regions (Figure 1 and Figure 2), the samples were sequenced and the resulting sequences aligned. Approximately $10 \%$ of sequences contained mutations between codons 377 and 594 in conserved regions of the UL97 gene, leading to amino acid changes (Table 1 and Figure 3). Of all the coding mutations observed, $20 \%(\mathrm{n}=2)$ were transversions [i.e., substitution of a purine $(\mathrm{A} / \mathrm{G})$ with a pyrimidine $(\mathrm{C} / \mathrm{T})]$ and $80 \%(n=8)$ were other types of substitutions (i.e., substitution of purines with purines or pyrimidines with pyrimidines).

Comparison revealed that $100 \%(n=82)$ of the obtained sequences harbored mutations, consisting of nucleotide substitutions leading to amino acid changes in two positions: one at position 1786, the first base of codon 596, GAG $\rightarrow$ AAG, leading to a glutamic acid to lysine alteration (E596K); and another at nucleotide position 1811 of codon $604, \mathrm{TCC} \rightarrow \mathrm{TTC}$, causing a serine to phenylalanine substitution (S604F). Given the high frequencies of these mutations in our samples, we compared them with 30 other sequences deposited in the GenBank database from countries including North America, Europe, and Asia; these mutations were not observed in any of the 30 other sequences.

In intragroup analysis of samples from patients with different forms of immune compromise, patients with HIV/ AIDS presented with a higher frequency $(\mathrm{n}=5 ; 62.5 \%)$ of point mutations leading to amino acid changes; transversion mutations were observed only in two samples in this group: $\mathrm{H}-100(\mathrm{C} \rightarrow \mathrm{G})$ and $\mathrm{H}-282(\mathrm{G} \rightarrow \mathrm{T})$. These samples exhibited two distinct genotypes encoding the amino acid changes $\mathrm{A} 377 \mathrm{G}$ and A378V in H-100 and A434F and M526I in H-282.

In the SLE patient group, only a single sample had a mutation leading to an amino acid change, while in the kidney transplant patient group, two samples had mutations causing amino acid substitutions, one of which corresponded to an alteration associated with resistance to ganciclovir, frequently described in literature.

Eleven genotypes leading to amino acid changes in the phosphotransferase chain of the UL97 gene were identified, located in different regions (Table 1). The amino acid changes were $\mathrm{A} 377 \mathrm{G}$ and $\mathrm{A} 378 \mathrm{~V}$ (regions III and IV); $\mathrm{A} 434 \mathrm{~F}$ and $\mathrm{A} 434 \mathrm{H}$ (region VI); G501L, N510S, V542I, and M526I (region VIII); and A594V, E596K, and S604F (region IX). The genotypes 


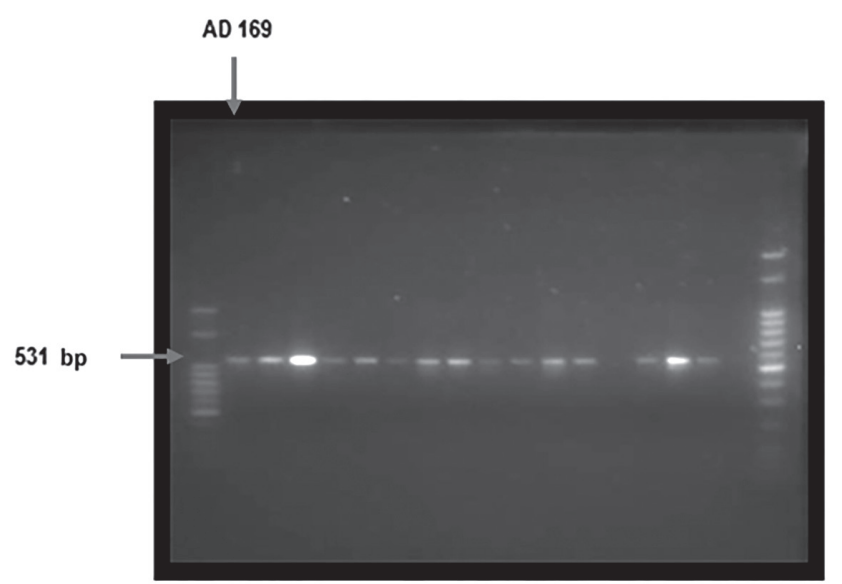

FIGURE 1: Electrophoresis of the 531-bp product amplified from the UL97 gene by nested-PCR reaction on a $1.5 \%$ agarose gel. The first well contains a 50bp molecular marker, the second well contains a positive control PCR product amplified from the AD169 strain, and the last well contains a 100bp molecular marker. The other lanes contain products from the amplification of the $531 \mathrm{bp}$ region of the UL97 gene by nested-PCR from representative clinical samples. The line 17 contains a negative control. In line 13 , there was no amplification, the sample was tested and applied in another gel not presented in this manuscript. bp: base pair; PCR: polymerase chain reaction.

encoding E596K and S604F were detected in 100\% of analyzed samples, including in the control AD169 laboratory strain.

Mutations leading to the substitutions A377G, A378V, A434H, M526I, N510S, G501L, and V542I were each present

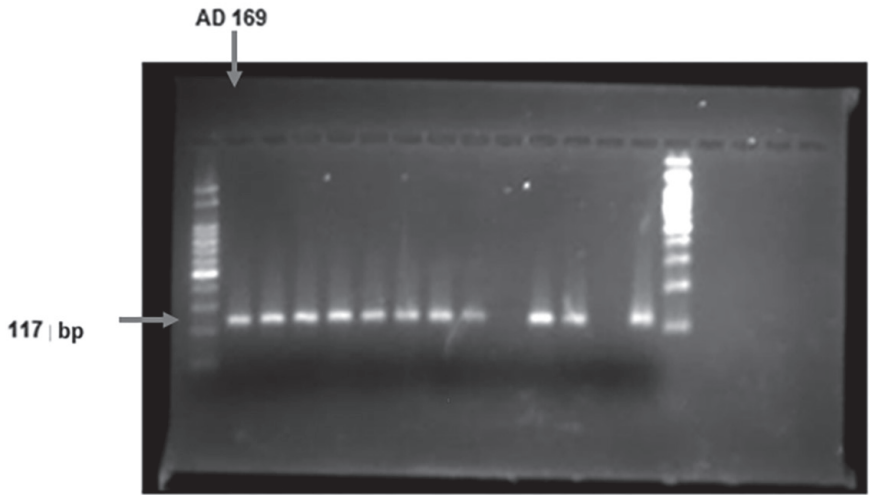

FIGURE 2: Electrophoresis of the 117-bp amplified products from the UL97 gene by nested-PCR on a $1.5 \%$ agarose gel. The first and last wells were loaded with 50 and $100 \mathrm{bp}$ molecular markers, respectively. The second well contains a positive control PCR product amplified from the AD169 strain, and the other wells contain products from the amplification of the $117 \mathrm{bp}$ region of the UL97 gene by nested-PCR from representative clinical samples. The negative control was not applied in this gel. Lanes 9 and 12 indicated no amplification; the samples were tested and amplified in another gel not presented in this manuscript. bp: base pair; PCR: polymerase chain reaction.

in one of five samples from patients with HIV, corresponding to $6.1 \%$ of all samples sequenced; the A434F genotype was observed in a single SLE patient sample and corresponded to $1.2 \%$ of all samples; N510S and A594V genotypes were each found in samples from patients receiving kidney transplants ( $2.4 \%$ of the 82 samples analyzed). The A594V genotype is associated with the antiviral resistance phenotype. The N510S genotype was present in two samples, one from an HIV carrier and the other from a kidney transplant patient.

TABLE 1: Mutations in the UL97 gene resulting in amino acid changes, in samples from immunocompromised patients from Belém-PA.

\begin{tabular}{|c|c|c|c|}
\hline Sample number* & Codon number & $\begin{array}{c}\text { Nucleotide change } \\
\text { (in context of triplet codon) }\end{array}$ & $\begin{array}{l}\text { Amino acid } \\
\text { change }\end{array}$ \\
\hline $\mathrm{H}-73$ & 501 & GAG $\rightarrow$ AAG & Glu $\rightarrow$ Lys \\
\hline $\mathrm{H}-100$ & 377 & $\mathrm{GCC} \rightarrow \mathrm{GGC}$ & $\mathrm{Ala} \rightarrow$ Gly \\
\hline $\mathrm{H}-100$ & 378 & $\mathrm{GCT} \rightarrow \mathrm{GTT}$ & $\mathrm{Ala} \rightarrow \mathrm{Val}$ \\
\hline H- 263 & 542 & $\mathbf{G T A} \rightarrow \mathbf{A T A}$ & Val $\rightarrow \| l e$ \\
\hline $\mathrm{H}-282$ & 434 & $\mathrm{CGT} \rightarrow \mathrm{CAT}$ & $\mathrm{Arg} \rightarrow \mathrm{His}$ \\
\hline T- 53.880 & 594 & $\mathrm{GCG} \rightarrow \mathrm{GTG}$ & $\mathrm{Ala} \rightarrow \mathrm{Val}$ \\
\hline SLE 43 & 434 & $\mathrm{CCG} \rightarrow \mathrm{CTG}$ & $\mathrm{Arg} \rightarrow \mathrm{Fen}$ \\
\hline
\end{tabular}

*Prefixes: H: HIV; T: kidney transplant; SLE: systemic lupus erythematosus. 


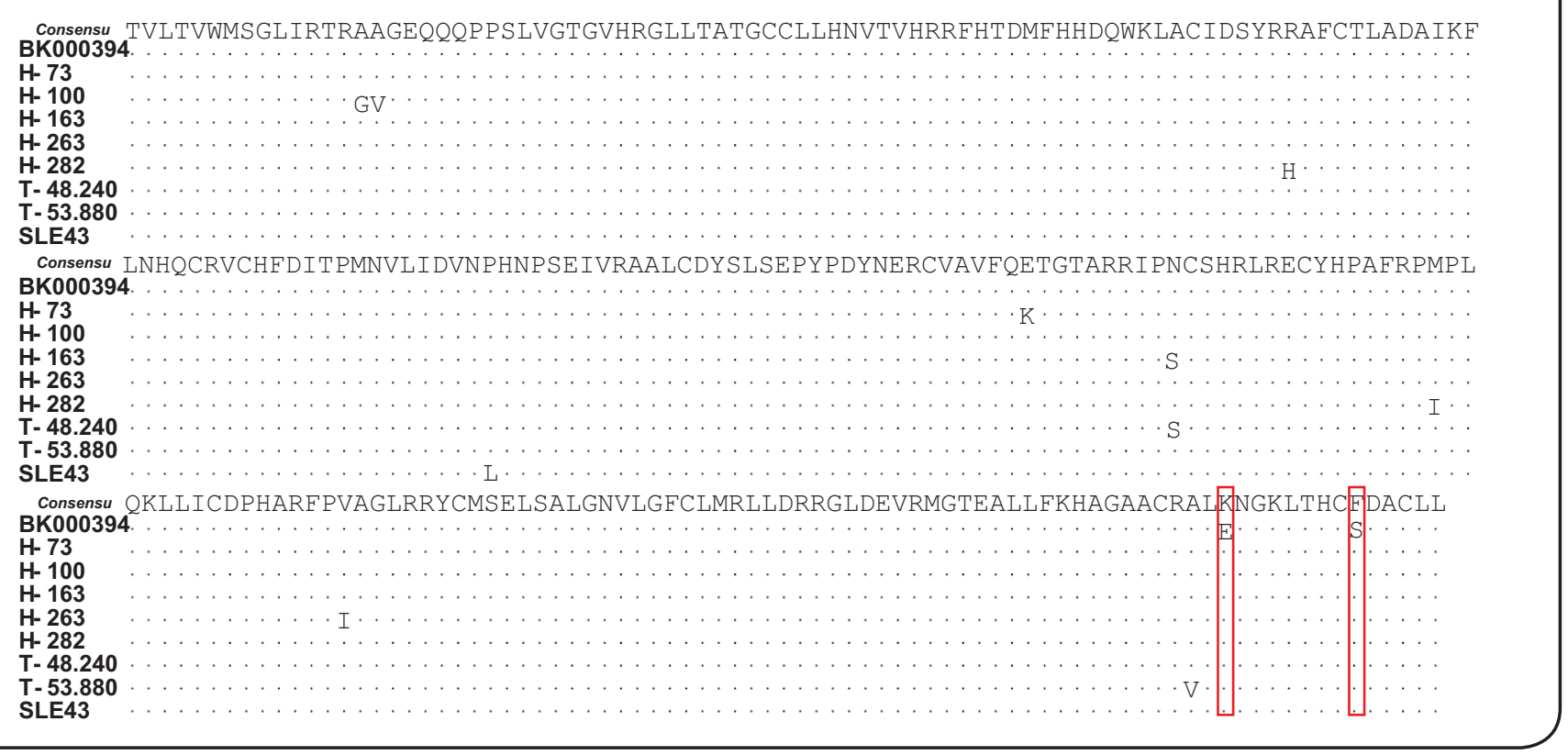

FIGURE 3: Mutations in UL97 leading to amino acid changes in the phosphotransferase chain. H: HIV; SLE: systemic lupus erythematosus; T: kidney transplant.

Based on the presence of mutations in the UL97 gene, the genotypic diversity among the groups appeared in a ratio of 7:2:1 for HIV: kidney transplant: SLE patients; therefore, these changes were more frequent in patients with HIV/AIDS.

\section{DISCUSSION}

Pyrosequencing analysis of the gene encoding viral phosphotransferase (UL97) detected a considerable number of silent mutations that did not affect the coding sequence, and eleven mutations leading to amino acid changes in the enzyme. The ratio of the high frequency of mutations found in patients with HIV/Aids was likely due to the discrepancy between the sample sizes of the groups. Future studies should be carried out with groups of equivalent size to validate the proportion of mutations found in the present study.

Among the amino acid-encoding changes, E596K and S604F, were detected in $100 \%(\mathrm{n}=82)$ of the sequenced samples, including the control AD169 strain. However, these two changes were not identified in the sequences from different studies available in the GenBank database, including sequences from Brazil and various other countries.

Nucleotide changes leading to two different substitutions at amino acid 596 (E596G and E596D) were described by Erice ${ }^{15}$ as associated with a change in codon 603 (C603Y). In this study, we identified a genotype different from that observed by Erice, encoding the substitution E596K. The E596K and S604F mutations have not previously been described in Brazil, either singly or in combination. This may be because only a small number of Brazilian studies on these mutations have been reported.
Other mutations introducing amino acid changes were observed less frequently and were present in eight samples (9.8\%), with 11 different genotypes. Among these, the A594V substitution was present in one individual undergoing kidney transplant. This mutation has previously been described by several researchers, including those from Brazil, as associated with resistance to ganciclovir, and is one of the most common mutations in the IX region of the $U L 97$ gene 1-4,6-8,23 $^{1}$.

Prolonged use ( $>3$ months) of ganciclovir may have induced the $\mathrm{A} 594 \mathrm{~V}$ resistance mutation in the kidney transplant patient, since according to their clinical history, the individual presented with recurrent infection and a high HCMV viral load $(\mathrm{M}=70,437.26$ copies $/ \mathrm{ml})$. According to Lurain and $\mathrm{Chou}^{1}$, factors such as a weakened host immune response, prolonged use of antiviral drugs, and continuous proliferation of HCMV may contribute to ganciclovir resistance.

Other mutations in different regions of the UL97 gene resulted in the following amino acid changes: $\mathrm{A} 377 \mathrm{G}$ and A378V (regions III and IV); A434F and A434H (region VI); and G501L, N510S, V542I, and M526I (region VIII). The N510S mutation was observed in a kidney transplant patient and was previously described by Erice in a clinical isolate from a patient with episodes of resistance to ganciclovir, together with deletion of codons $591-594^{15}$. Of the other mutations described in this study, only those leading to changes at positions 594, 596, and 510 , have previously been described in the literature.

Mutations detected in this study that led to amino acid substitutions in the IX region of the UL97 gene (A594V, E596K, and S604F) may affect the activity of the pUL97 enzyme, since this region corresponds to the binding site of the phosphotransferase to the viral DNA chain ${ }^{14}$. 
The detection of mutations leading to amino acid substitutions in the samples of the immunocompromised patients who volunteered to participate in this study may be associated with either the intermittent use of antiviral drugs due to recurrent HCMV infections or the recombination of viral strains. We hypothesize that the latter may have occurred, particularly in two of the samples from patients with HIV/AIDS (H-100 and $\mathrm{H}-282$ ), since more than one mutation was identified in these individuals: A377G/A378V and A434F/M526I, respectively. The emergence of more than one resistance genotype in a sample from a single patient is associated with recombination between wild-type and mutant strains, due to the occurrence of re-infection by an exogenous strain ${ }^{21,24-26}$. This may be due to use of antiviral drugs (possibly administered at inadequate doses for prolonged periods), high viral load, use of immunosuppressors, and/or weak immunological response of the patient. It was not possible to include data concerning antivirals due to insufficient information in patient records. Opportunist behavior due to latency and viral HCMV reactivation may lead to a high number of reinfections, necessitating the use of drugs for their control. Therefore, further studies are required, including the combined analyses of genes UL97 and UL54 DNA polymerase, to detect mutations associated with HCMV resistance to antiviral drugs to demonstrate the need for an increase in the variety and availability of drugs used to treat viral infections in the public health care network in Brazil.

\section{Acknowledgements}

We would like to thank all patients and professionals at the Evandro Chagas Institute and Ophyr Loyola, Jean Bitar, and João de Barros Barreto University Hospital, who contributed to the study.

\section{Financial support}

This work was supported by Instituto Evandro Chagas/Secretaria de Vigilância em Saúde/Ministério da Saúde (IEC/SVS/MS) and Fundação Amazônia Paraense de Amparo à Pesquisa (FAPESPA).

\section{Conflict of interest}

The authors declare that there is no conflict of interest.

\section{REFERENCES}

1. Lurain NS, Chou S. Antiviral drug resistance of human cytomegalovirus. Clin Microbiol Rev. 2010;23(4):689-712.

2. Erice A, Gil-Roda C, Pérez JL. Antiviral susceptibilities and analysis of UL-97 and DNA polymerase sequences of clinical cytomegalovirus isolates from immunocompromised patients. J Infect Dis Med. 1997;175(5):1087-92.

3. Chou S, Lurain NS, Weinberg A, Cai GY, Sharma PL, Crumpacker CS. Interstrain variation in the human cytomegalovirus DNA polymerase sequence and its effect on genotypic diagnosis of antiviral drug resistance. Adult AIDS Clinical Trials Group CMV Laboratories. Antimicrob Agents Chemother. 1999;43(6):1500-2.

4. Chou S, Waldemer RH, Senters AE, Michels K, Kemble GW, Miner $\mathrm{RC}$, et al. Cytomegalovirus UL97 phosphotransferase mutations that affect susceptibility to ganciclovir. J Infect Dis. 2002;185(2):162-9.

5. Gilbert C, Boivin G. Human cytomegalovirus resistance to antiviral drugs. Antimicrob Agents Chemother. 2005;49(3):873-83.
6. Boivin G, Goyette N, Gilbert C, Covington E. Analysis of cytomegalovirus DNA polymerase (UL54) mutations in solid organ transplant patients receiving valganciclovir or ganciclovir prophylaxis. J Med Virol. 2005;77(3):425-9.

7. Nogueira E, Ozaki KS, Tomiyama H, Granato CFH, Camara NOS, Pacheco-Silva A. The emergence of cytomegalovirus resistance to ganciclovir therapy in kidney transplant recipients. Int Immunopharmacol. 2006;6(13):2031-7.

8. Drew LW. Cytomegalovirus resistance testing: pitfalls and problems for the clinician. Clin Infect Dis. 2010;50(5):733-6.

9. Tatarowicz WA, Lurain NS, Thompson KDA. ganciclovirresistant clinical isolate of human Cytomegalovirus exhibiting cross-resistance to other DNA polymerase inhibitors. J Infect Dis. 1992;166(4):904-7.

10. Smith IL, Cherrington JM, Jiles RE, Fuller MD, Freeman WR, Spector SA. High-level resistance of Cytomegalovirus to ganciclovir is associated with alterations in both the UL97 and DNA polymerase genes. J Infect Dis 1997;176(1):69-77.

11. Chou S. Contrasting drug resistance phenotypes resulting from cytomegalovirus DNA polymerase mutations at the same exonuclease locus. J Infect Dis. 2008;43(1):107-9.

12. Carraro E, Granato CFH. Genotipagem do cytomegalovirus humano para pesquisa de resistência aos antivirais em transplantados renais. J Bras Patol Med Lab. 2004;40(1):11-14.

13. Silva DL, Medeiros RF, Moraes MM, Santo SF. Restriction enzyme analysis of the human cytomegalovirus genome in specimens collected from immunodeficient patients in Belém, State of Pará, Brazil. Rev Soc Bras Med Trop. 2011;44(5):551-4.

14. Göhring K, Hamprecht K, Jahn G. Antiviral drug and multidrug resistance in cytomegalovirus infected SCT patients. Comput Struct Biotechnol J. 2015;(13):153-9.

15. Erice A. Resistance of human cytomegalovirus to antiviral drugs. Clin Microbiol Rev. 1999;12(2):286-97.

16. Schleiss MR. Antiviral therapy of congenital cytomegalovirus infection. Semin Pediatr Infect Dis. 2004;16(1):50-9.

17. Wolf DG, Smith IL, Lee DJ, Freeman WR, Flores-Aguilar M, Spector SA. Mutations in human cytomegalovirus UL97 gene confer clinical resistance to ganciclovir and can be detected directly in patient plasma. J Clin Invest. 1995;95(1):257-63.

18. Gilbert C, Bestman-Smith J, Boivin G. Resistance of herpesviruses to antiviral drugs: clinical impacts and molecular mechanisms. Drug Resist Updat. 2002;5(2):88-114.

19. Boeckh M, Ljungman P. How we treat cytomegalovirus in hematopoietic cell transplant recipients. Blood. 2009;113(23):5711-9.

20. Sanger F, Nicklen S, Coulson AR. DNA sequencing with chainterminating inhibitors. Proc Natl Acad Sci USA. 1977;74(12):5463-7.

21. Boivin G, Chou S, Quirk MR, Erice A, Jordan MC. Detection of ganciclovir resistance mutations and quantitation of cytomegalovirus (CMV) DNA in leucocytes of patients with fatal disseminated CMV disease. J Infect Dis. 1996;173(3):523-8.

22. Margulies M, Egholm M, Altman WE, Said A, Joel SB, Lisa AB, et al. Genome sequencing in microfabricated high-density picolitre reactors. Nature. 2005;437(7057):376-80.

23. Chou S, Erice A, Jordan MC, Vercellotti GM, Michels KR, Talarico CL, et al. Analysis of the UL97 phosphotransferase coding sequence in clinical cytomegalovirus isolates and identification of mutations conferring ganciclovir resistance. J Infect Dis. 1995;171(3):576-83.

24. Lymaye A. Ganciclovir-resistant cytomegalovirus in organ transplant recipients. Clin Infect Dis. 2002;35(7):866-73.

25. Rodriguez J, Casper K, Smallwood G, Stieber A, Fasola C, Lehneman J, et al. Resistance to combined ganciclovir and foscarnet therapy in a liver transplant recipient with possible dual-strain cytomegalovirus coinfection. Liver Transpl. 2007;13(10):1396-400.

26. Madi N, Al-Nakib W, Pacsa A. Does cytomegalovirus develop resistance following antiviral prophylaxis and treatment in renal transplant patients in Kuwait? Adv Virol. 2011;2011:260561. 